\title{
Large bowel obstruction from advanced prostate cancer in a 41-year-old: the role of genetic testing
}

\author{
JB Jonosky, ${ }^{1,2,3}$ (i) N de Villiers, ${ }^{4}(\mathbb{D})$ R Wadee, ${ }^{5,6}$ (D) F Chinegwundoh, ${ }^{7}$ (i) A Adam ${ }^{1,2,3}$ \\ ${ }^{1}$ Department of Urology, Helen Joseph Hospital, South Africa \\ ${ }^{2}$ Department of Paediatric Urology, Rahima Moosa Mother \& Child (Coronation) Hospital, South Africa \\ ${ }^{3}$ Division of Urology, Department of Surgery, Faculty of Health Sciences, University of the Witwatersrand, South Africa \\ ${ }^{4}$ AMPATH Genetics, South Africa \\ ${ }^{5}$ Division of Anatomical Pathology, University of the Witwatersrand, South Africa \\ ${ }^{6}$ National Health Laboratory Services (NHLS), South Africa \\ ${ }^{7}$ Department of Urology, Barts Health NHS Trust, United Kingdom
}

Corresponding author, email: jaxwallington@icloud.com

\begin{abstract}
Summary
We report a rare presentation of an advanced prostate cancer (Gleason 10) in a 41-year-old man with complete large bowel obstruction relieved by loop colostomy. His young age prompted a search for a cause and genetic testing confirmed chromosome 10 PTEN deletion. His immediate family members were screened and counselled appropriately. This case outlines the role of genetic testing in young males with prostate cancer.
\end{abstract}

\section{Case report}

A 41-year-old male presented to the emergency department with a history of constipation for two days. His vitals were stable. The abdomen was distended and tender. On digital rectal examination, the ano-rectum was noted to be stenosed and an irregular mass was palpated. A plain film abdominal radiograph showed evidence of bowel obstruction (Figure 1). The PSA was found to be elevated at $67 \mathrm{ng} / \mathrm{mL}$ (age related upper limit of normal $2.5 \mathrm{ng} / \mathrm{ml}$ ).

The patient was then managed by general surgery as a possible malignant bowel obstruction, and an emergency diverting colostomy was performed. The surgeon noted the tumour to be almost completely obstructing the rectum and colonoscopy was not possible. Initial histology from biopsies taken from the rectum confirmed an adenocarcinoma that on further staining, was found to be of prostatic rather than bowel origin. A formal trans-rectal ultrasound guided prostate (TRUS) biopsy confirmed prostate adenocarcinoma. The Gleason major and minor scores were each 5, giving a combined score of 10. A magnetic resonance imaging (MRI) scan (Figure 2) showed a mass arising from the prostate breaching the capsule and invading into the rectum. No evidence of metastasis was seen on the MRI sections examined. A bone scan showed no evidence of osteoblastic metastasis.

Due to his young age and T4 disease, genetic studies were undertaken and a deletion of exon 1 of the PTEN gene was detected. No pathogenic or likely pathogenic sequence variants were detected in the coding regions of the BRCA1 or BRCA2 genes. Tumour slides from the patient were received and multiplex ligation-dependent probe amplification

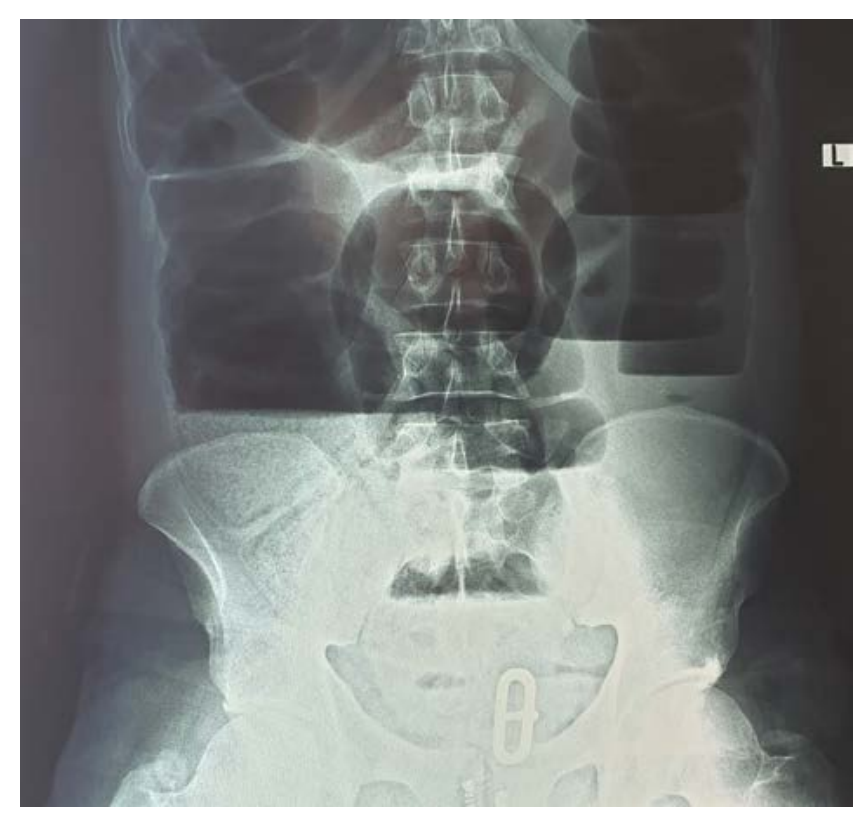

Figure 1: Plain film abdominal X-ray showing multiple air-fluid levels and paucity of gas distally, indicating distal large bowel obstruction

(MLPA) analysis of the PTEN gene was performed using the MRC Holland Kit P225-PTEN. Peripheral blood from the patient was screened for sequence variants in the coding and immediate intronic regions of the BRCA1 and BRCA2 genes, using bidirectional sequencing of PCR (polymerase chain reaction) generated products. The DNA sequence and MLPA data was analysed electronically. 


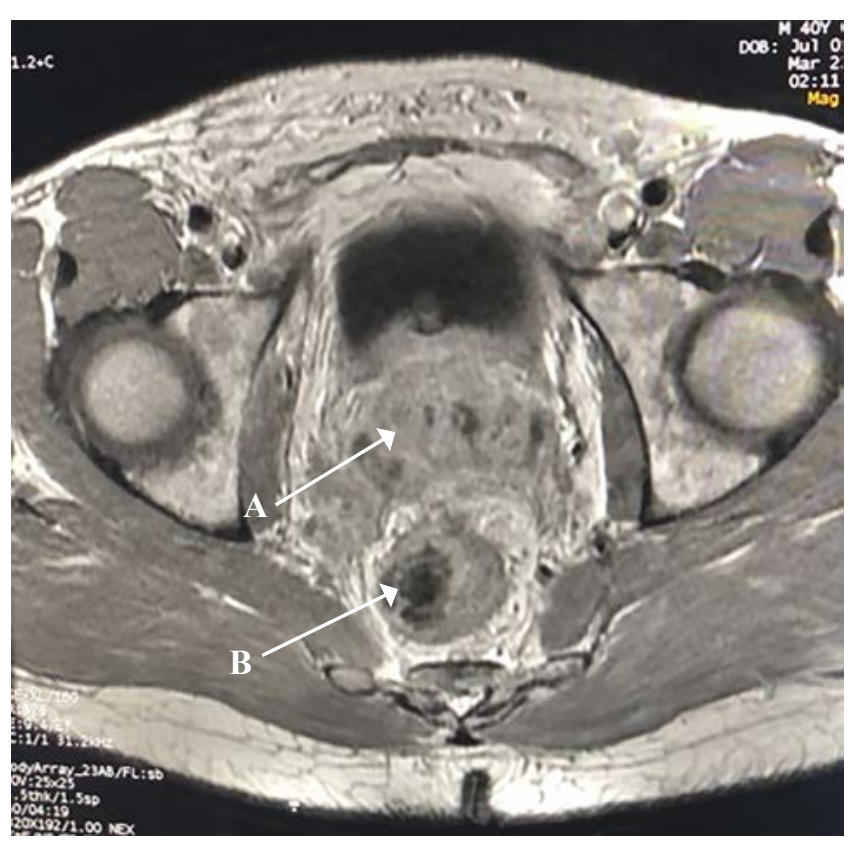

Figure 2: MRI shows a heterogeneous mass arising from the prostate (A), with irregular lateral margins, invading into the anterior wall of the rectum $(B)$

The patient remained with a colostomy and was then placed on maximum androgen deprivation therapy (ADT). Due to continued local invasion, he developed renal failure from bilateral hydronephrosis. Despite percutaneous nephrostomies, he died one year later of progressive renal failure. Two of the patient's brothers were counselled on the likelihood of developing prostate cancer early and the implications of this genetic finding for themselves and their families. They were screened using digital rectal examination and PSA testing and they were found to be negative thus far for prostate cancer. They were also offered the opportunity to test for his specific PTEN mutation but were unable to return for testing due to social and economic reasons.

\section{Discussion}

In our review of the literature, large bowel obstruction requiring diversion though rare $(1-4 \% \text { of cases })^{1}$ most commonly occurs in elderly males with long-standing history of known metastatic prostate carcinoma and high PSA values at the time of gastrointestinal (GIT) complaints. Where locally invasive disease is present, the seminal vesicles and the base of the bladder are typical sites involved since prostate carcinoma's mechanism of spread is via the venous and lymphatic channels superiorly rather than via direct posterior extension to the rectum which is further protected by Denonvilliers Fascia. ${ }^{1-3}$ Bowel obstruction secondary to prostate carcinomas can be due to direct invasion producing a stenosing annular lesion, as in this case, a large mass protruding into the rectum, a separate recto-sigmoid metastasis, or concomitant adenocarcinomas of the prostate and rectum. ${ }^{4}$

The genetic basis of prostate cancer has become an increasingly important point of discussion owing to a large heritable contribution in about $58 \%$ of cases. ${ }^{5}$ As shown in this case, the tumour suppression gene PTEN was deleted on chromosome 10. Such a deletion has been shown to be important in many malignancies including prostate cancer. ${ }^{5}$ Though it has been documented that the effect of this gene can vary from tumour initiation through to metastatic progression, the prognostic value of the PTEN deletion remains unclear. ${ }^{5}$ The South African Prostate Cancer Study (SAPCS) has shown that South African men of African and mixed ancestry present with a two-fold increase in the frequency of aggressive disease when compared to African Americans. ${ }^{6}$ While socio-economic, lifestyle and inherited basis are contributing factors, it is increasingly recognised that genetic factors likely play a significant role in this difference in tumor biology. ${ }^{6}$

The Philadelphia Prostate Cancer Consensus Conference held in 2017 attempted to identify genes to be screened and guidelines for patient identification and screening for genetic factors. The Philadelphia consensus identified genes BRCA 1 and 2, HOXB13, DNA MMR and ATM as important because they increase a patient's risk of developing prostate cancer anywhere from two- to five-fold as well as placing the patient at greater likelihood of developing castrate resistant prostate cancer. ${ }^{5}$ In the consensus, it was suggested that if patients show positive genetic results their family should then be screened for prostate cancer ten years earlier than the initial presentation of their diagnosed family member or at the age of forty. ${ }^{5}$ However, these genetic variations are based on European studies and genetic findings. In this case report, we highlight the importance of a PTEN mutation on chromosome 10 as was confirmed in other studies involving whole genome sequencing, where small somatic variants (such as PTEN) were found to be approximately four times higher in South African men with high risk prostate cancer than in the European population. ${ }^{6}$ These small oncogenic drivers present in this population group appear to contribute to a higher tumour mutational burden. ${ }^{6}$ The identification of these and other smaller oncogenic drivers are important as they can predict future responses to targeted immunotherapy. ${ }^{6}$

\section{Acknowledgements}

The authors would like to thank the staff at National Health Laboratory Services (NHLS) as well as AMPATH Laboratories for genetic assay testing performed without cost to the patient or institution.

\section{Conflict of interest}

The authors declare no conflict of interest.

\section{Ethical approval}

Local institutional ethics clearance was attained with Human Research Ethics Committee (Medical). Certificate clearance number: M190130 (2202/2019).

\section{ORCID}

JB Jonosky (D) https://orcid.org/0000-0002-9102-5363

$\mathrm{N}$ de Villiers (D) https://orcid.org/0000-0002-8059-9054 R Wadee (iD https://orcid.org/0000-0002-5981-4450 F Chinegwundoh (iD https://orcid.org/0000-0002-7466-7225 A Adam (D) https://orcid.org/0000-0001-9069-3282

\section{REFERENCES}

1. Kabeer M, Lloyd-Davies E, Maskell G, Hohle R, Mathew J. Metastatic prostate cancer masquerading clinically and radiologically as a primary caecal carcinoma. World J Surg Oncol. 2007;5:2. https://doi.org/10.1186/1477-7819-5-2. PMID: 17207288. 
2. Aigen A, Schapira H. Metastatic carcinoma of prostate and bladder causing intestinal bowel obstruction. Urology. 1983;21(5):464-6. https://doi.org/10.1016/0090-4295(83)90 041-9. PMID: 6857883.

3. Ruggiero R, Chang H. Rectal involvement by carcinoma of the prostate. Am J Gastroenterol. 1986;81(5):372-4. PMID: 3486587.

4. Davies A. Prostatic carcinoma presenting as acute obstruction of large bowel. Cancer. 1967;20(6):1035-37. https://doi. org/10.1002/1097-0142(196706)20:6\%3C1035::AID-CNCR 2820200615\%3E3.0.CO;2-9. PMID: 6026468.
5. Veda G, Knudsen K, Kelly W, et al. Role of genetic testing for inherited prostate cancer risk: Philadelphia Prostate Cancer Consensus Conference 2017. J Clin Oncol. 2018;36(4):414-24 https://doi.org/10.1200/JCO.2017.74.1173. PMID:29236593.

6. Jaratlerdsiri W, Chan E, Gong T, et al. Whole genome sequencing reveals elevated tumour mutational burden and initiating driver mutations in African men with treatment naïve high-risk prostate cancer. Cancer Res. 2018;78(24):6736-46. https://doi.org/10.1158/0008-54. PMID: 30217929. 